\title{
Mirror Movement-Like Defects in Startle Behavior of Zebrafish $d c c$ Mutants Are Caused by Aberrant Midline Guidance of Identified Descending Hindbrain Neurons
}

\author{
Roshan A. Jain, ${ }^{1}$ Hannah Bell, ${ }^{1}$ Amy Lim, ${ }^{2,3}$ Chi-Bin Chien, ${ }^{2 \dagger}$ and Michael Granato ${ }^{1}$ \\ ${ }^{1}$ Department of Cell and Developmental Biology, University of Pennsylvania, Perelman School of Medicine, Philadelphia, Pennsylvania 19104, ${ }^{2}$ Department \\ of Neurobiology and Anatomy, University of Utah, Salt Lake City, Utah 84132, and ${ }^{3}$ Molecular Medicine Program, University of Utah, Salt Lake City, Utah \\ 84112
}

Mirror movements are involuntary movements on one side of the body that occur simultaneously with intentional movements on the contralateral side. Humans with heterozygous mutations in the axon guidance receptor DCC display such mirror movements, where unilateral stimulation results in inappropriate bilateral motor output. Currently, it is unclear whether mirror movements are caused by incomplete midline crossing and reduced commissural connectivity of $D C C$-dependent descending pathways or by aberrant ectopic ipsilateral axonal projections of normally commissural neurons. Here, we show that in response to unilateral tactile stimuli, zebrafish $d c c$ mutant larvae perform involuntary turns on the inappropriate body side. We show that these mirror movement-like deficits are associated with axonal guidance defects of two identified groups of commissural reticulospinal hindbrain neurons. Moreover, we demonstrate that in $d c c$ mutants, axons of these identified neurons frequently fail to cross the midline and instead project ipsilaterally. Whereas laser ablation of these neurons in wild-type animals does not affect turning movements, their ablation in $d c c$ mutants restores turning movements. Thus, our results demonstrate that in $d c c$ mutants, turns on the inappropriate side of the body are caused by aberrant ipsilateral axonal projections, and suggest that aberrant ipsilateral connectivity of a very small number of descending axons is sufficient to induce incorrect movement patterns.

Key words: axon guidance; DCC; movement disorders; zebrafish

\section{Introduction}

Right/left body coordination is disrupted in mirror movement disorder (MMD), where voluntary unilateral hand or finger movements are accompanied by involuntarily synchronous movements on the opposite side of the body (Galléa et al., 2011). Mirror movements, although transiently observed in normal human early development, persist through adulthood in genetically diverse familial neurological disorders (Peng and Charron, 2013). Congenital "essential MMD," where highly penetrant mirror movements are observed without other symptoms, has been linked to haploinsufficient Deleted in Colorectal Carcinoma (DCC) mutations (Srour et al., 2010; Depienne et al., 2011). DCC

\footnotetext{
Received June 5, 2013; revised Jan. 13, 2014; accepted Jan. 16, 2014.

Author contributions: R.A.J. and M.G. designed research; R.A.J. and H.B. performed research; A.L. and C.-B.C. contributed unpublished reagents/analytic tools; R.A.J., H.B., and M.G. analyzed data; R.A.J. and M.G. wrote the paper.

This work was supported by National Institutes of Health Ruth L. Kirschstein National Research Service Award 5F32NS065637 to R.A.J. and National Institutes of Health Grants MH092257 and HD37975 to M.G. We thank Olav Olsen, Marc Tessier-Lavigne, Puneet Deng, Jesse Issacman-Beck, and Alvaro Gutiérrez-Uzquiza for helpful technical advice and reagents for cell culture experiments.

The authors declare no competing financial interests.

${ }^{\dagger}$ Deceased.

Correspondence should be addressed to Dr. Michael Granato, 421 Curie Blvd, 1210 BRB II/III, Philadelphia, PA 19104. E-mail: granatom@mail.med.upenn.edu.

DOI:10.1523/JNEUROSCI.2420-13.2014

Copyright $\odot 2014$ the authors $\quad 0270-6474 / 14 / 342898-12 \$ 15.00 / 0$
}

encodes a Netrin receptor, which guides neuronal processes across the CNS midline, consistent with its behavioral role in left/right movement coordination (Keino-Masu et al., 1996; Serafini et al., 1996; Fazeli et al., 1997). DCC's conserved role in bilateral motor coordination has been demonstrated in Dcc and Netrin knock-out mice, where isolated spinal cords revealed defects in left/right alternating spinal activity (Rabe et al., 2009; Rabe Bernhardt et al., 2012). Strikingly, mice carrying the hypomorphic Dcc ${ }^{\text {kanga }}$ allele are viable and display synchronous rather than alternating hindlimb movements, although it is unclear whether local spinal disruptions or inappropriate descending inputs produce this (Finger et al., 2002; Rabe Bernhardt et al., 2012). Thus, despite a clear role for DCC in commissural axon guidance, distinguishing the specific neuronal deficits causing the behavioral disruptions has been difficult (Peng and Charron, 2013).

Several overlapping models have been proposed to explain the neuronal basis of human mirror movement behavior resulting from DCC disruption. Loss of commissural inhibitory axonal connections of the corpus callosum might produce inappropriate bilateral activation of the sensorimotor cortex (Galléa et al., 2011; Lepage et al., 2012; Fothergill et al., 2013). Alternatively, inappropriate ipsilateral targeting of a subset of corticospinal tract axons could cause the behavioral deficits (Peng and Charron, 2013), consistent with unilateral motor cortex stimulation in DCC pa- 
tients producing bilateral motor activation (Cincotta et al., 2003; Depienne et al., 2011). The relative causal contributions of reduced left/right neuronal connectivity versus ectopic ipsilateral connectivity to the aberrant behavioral pathology has remained unclear.

Here, we take advantage of the well-characterized neuroanatomy of the zebrafish hindbrain to probe the role of identified neurons in the etiology of mirror movement-like behavioral deficits in $d c c$ mutants. Specifically, we show that zebrafish spaced out (spo) mutants carry $d c c$ mutations, including a single amino acid substitution disrupting Netrin binding. Millisecond-resolution analyses demonstrate that $d c c$ mutants perform involuntary turns on the inappropriate body side after localized touch stimulation, and these behavioral defects correlate with aberrant ipsilateral axonal projections of $\mathrm{MiD} 2 \mathrm{~cm}, \mathrm{MiD} 3 \mathrm{~cm}$, and $\mathrm{MiD} 3 \mathrm{cl}$ reticulospinal neurons. Although selectively ablating these commissural neurons does not affect touch-evoked responses in wild-type animals, MiD2/MiD3 neural ablation in $d c c$ mutants restricts involuntary turns back to the appropriate body side. Together, our data demonstrate that in zebrafish $d c c$ mutants, it is not the lack of hindbrain commissural connectivity, but rather a small subset of aberrant ipsilaterally misprojecting MiD2/MiD3 reticulospinal hindbrain neurons, that is sufficient to activate movements on the inappropriate body side.

\section{Materials and Methods}

Zebrafish lines and maintenance. All lines were crossed into and maintained in the wild-type Tüpfel Long Fin strain, with the exception of the mapping cross, which used the polymorphic WIK-L11 strain (Rauch et al., 1997). The $s p o^{t m 272 b}$ and $s p o^{t s 239}$ mutants were generated in the Tübingen background (Granato et al., 1996). The $d c c^{z m 130198} 5.2 \mathrm{~kb}$ retroviral insertion allele was generated by Znomics (Jao et al., 2008). We have previously described the $\mathrm{Tg}(\mathrm{T} 2 \mathrm{KSAG}) j 1229 a$ GFP enhancer trap line, hereafter referred to as simply j1229a (Burgess et al., 2009). In all $d c c$ mutant analyses, all mutant, sibling, and control larvae were raised together at $21^{\circ} \mathrm{C}-24^{\circ} \mathrm{C}$, as neural and behavioral phenotypes were more severe and penetrant at this temperature range than at warmer temperatures. Unless otherwise specified, spaced out/dcc mutant data presented used the $s p o / d c c^{t m 272 b}$ allele. Larval zebrafish of either sex were used for all experiments, in accordance with Institutional Animal Care and Use Committee regulatory standards.

Mapping, sequencing, and genotyping spaced out/dcc mutants. Bulk segregant mapping was performed on $s p o^{t s 239}$ as previously described (Burgess et al., 2009), using a pool of 25 behaviorally mutant larvae and a pool of 25 behaviorally normal siblings. The linked SSLP markers z24994 (GenBank \#G47488.1) and z23466 (GenBank \#G45410.1) were examined by PCR, and mapped to the zebrafish genome assembly $\mathrm{Zv} 9$ (GCA_000002035.2).z24994 mapped 1182 bp downstream of the first coding exon of $d c c$ and z23466 mapped $778 \mathrm{bp}$ upstream of the third coding exon of $d c c$. The $d c c^{z m 130198}$ allele carries a retroviral insertion of $\sim 5.2 \mathrm{~kb}$ in the $5^{\prime} \mathrm{UTR}$ of $d c c$, located $106 \mathrm{bp}$ upstream of the start codon (see Fig. 1H) (Jao et al., 2008).

Full-length $d c c$ cDNA was amplified from 6 days postfertilization (dpf) Tüpfel Long Fin and behaviorally mutant larval RNA using SuperScriptII reverse transcriptase (Invitrogen) and Phusion polymerase (NEB). Genotyping for the $s p o^{t m 272 b}$ allele was performed by amplifying genomic DNA using a pair of dCAPS (Neff et al., 2002) genotyping primers: $5^{\prime}$-CCCAGCTCTCATTACGTGG-3' and $5^{\prime}$-GTCTGTCATGGATCTG GTG-3', followed by Hinfl digestion, which cuts only the mutant product. Genotyping for the $d c c^{z m 130198}$ allele was performed by amplifying genomic DNA using a mixture of the following 3 primers: (dcc5'UTR-F1) 5'-GCGCAGCTGTCTGTCAGTAG-3', (DCCzm-130198) 5'-CGCAGATCTGTGCGTAGGAGAGC-3', and (DCCzm-5'LTR) 5'GACGCAGGCGCATAAAATCAGTC-3', which together amplify a 203 bp band for the wild-type allele and an $\sim 700$ bp band for the $d c c^{2 m 130198}$ allele. Quantitative RT-PCR was performed using the SYBR Green Jump-
Start qPCR mix on total cDNA generated from 3 or 4 independent pools of 3 embryos. For $50 \mathrm{hpf} d c^{2 m 130198}$ mutant samples, total RNA and genomic DNA was extracted from individuals using TRIzol reagent (Invitrogen), and only homozygous wild-type or $d c c^{z m 130198}$ individuals were used for analysis.

The reference DCC protein sequences used in alignments of Figure $1 C$ were as follows: D. melanogaster Frazzled isoform A (NP_523716.2), C. elegans Unc40 (NP_491664.1), M. musculus DCC (NM_007831.3), and H. sapiens DCC (NM_005215.3).

Netrin binding analysis. Full-length zebrafish $d c c$ and $d c c^{t m 272 b}$ coding sequences were PCR amplified without a stop codon, fused at the C terminus to a Gly-Gly linker followed by EGFP lacking the initial Met codon, and cloned between the EcoRI and SnaBI sites of the pCS2+ expression vector. The MuSK-GFP expression construct (SV1 isoform) was previously described (Gordon et al., 2012). Cos-7 cells were transiently transfected in parallel with DNA using FuGENE 6 (Promega). At $48 \mathrm{~h}$ after transfection, cells were washed $2 \times$ with Netrin Binding Buffer (HBSS, pH 7.4, supplemented with $5 \mathrm{~mm} \mathrm{CaCl}_{2}, 1 \mathrm{~mm} \mathrm{MgCl}_{2}, 0.2 \% \mathrm{BSA}$, $1 \mathrm{mg} / \mathrm{ml} \mathrm{NaN}_{3}, 1 \mu \mathrm{g} / \mathrm{ml}$ heparin), then overlaid with $5 \mu \mathrm{g} / \mathrm{ml} \mathrm{recombi-}$ nant human FLAG-Netrin-1 (Enzo Life Sciences) in Netrin Binding Buffer on ice for $90 \mathrm{~min}$. Cells were washed $5 \times$ with cold Netrin Binding Buffer and then fixed 15 min with $4 \%$ paraformaldehyde/PBS, and stained with rabbit anti-GFP (1:500, Clontech) and mouse anti-FLAG M2 (1:100, Sigma), followed by Alexa-488-goat-anti-rabbit (1:1000, Invitrogen), Alexa594-goat-anti-mouse (1:1000, Invitrogen), and DAPI, then mounted in Vectashield (Vector Laboratories). Cells were imaged in the same session on a Zeiss LSM710 confocal microscope using identical acquisition settings. Background-corrected total cell fluorescence of GFP-positive cells was calculated using FIJI (Schindelin et al., 2012), and all cells exceeding a 80,000 minimum GFP corrected total cell fluorescence threshold were analyzed for Netrin-FLAG binding by measuring background-corrected total immunofluorescent intensity of anti-FLAG staining (Burgess et al., 2010).

Behavioral analysis of intact larvae. Acoustic stimuli were delivered to free-swimming d5-d7 larvae housed in individual wells of a $4 \times 4$ grid and recorded from above at $1000 \mathrm{fps}$ as previously described (Wolman et al., 2011). Larvae received 20 nondirectional acoustic stimuli. Tactile stimuli were manually delivered using a short piece of nylon fishing line attached to a glass capillary tube handle. Each larva received at least 5 stimuli per side for head and tail stimuli, for a total of 20-30 tactile stimuli per larva. Automated analysis of larval movement kinematics (response frequency, latency, turning angle, body curvature) was performed using the FLOTE software package (Burgess and Granato, 2007b). Counterbend performance and direction were scored manually from the video, blind to the genotype of the larvae. Responses where bending direction was ambiguous due to larval orientation were not included in the analyses. Spontaneous swimming movements were recorded for larvae in $8 \mathrm{~s}$ blocks and scored manually for left/right alternating tail bends. Spontaneous tail curvature was calculated using FLOTE. All larvae were individually genotyped after behavioral testing.

Statistical analysis. Statistical comparison of behaviors between groups was performed with GraphPad Prism v5.0d software using the two-tailed Student's $t$ test with Welch's correction for unequal variance, unless otherwise specified. Where multiple kinematic parameters were analyzed for a given dataset, Bonferroni correction was applied to $p$ values. Mauthner array axonal phenotypes were analyzed using a 1-tailed Fisher exact test.

Immunofluorescent hindbrain labeling. The $60-72 \mathrm{hpf}$ embryos for hindbrain reticulospinal immunofluorescence were raised in $0.2 \mathrm{~mm}$ phenylthiourea/E3 from $24 \mathrm{hpf}$ to prevent pigmentation, fixed with $2 \%$ trichloroacetic acid/PBS, and stained with anti-intermediate neurofilament M ( $\alpha$ RMO44) as described by Waskiewicz et al. (2001). Tails of stained larvae were retained during mounting, imaging was performed blind to larval genotype, and tails were used to genotype all individuals following commissural axon scoring. Commissural axon projections were manually followed and scored through each $z$-stack. Both MiD3cl neurons were not always present and/or stained in all larvae, regardless of wild-type/heterozygous/mutant genotype, so only those neurons with unambiguously identified cell bodies were scored (6-8 total Mauthner 
array neurons per larva). GFP signal of $j 1229 a$ was used as an additional guide in the $d c c^{t m 272 b}$ background. Because $\alpha \mathrm{RMO} 44$ signal alone was also sufficient to locate all present Mauthner/MiD2 $\mathrm{cm} / \mathrm{MiD} 3 \mathrm{~cm} / \mathrm{MiD} 3 \mathrm{cl}$ cell bodies in all of these cases, $\alpha$ RMO44 staining alone was used in scoring neurons in the $d c c^{z m 130198}$ background. Larval hindbrains at $6 \mathrm{dpf}$ were fixed overnight in sweet fix ( $4 \%$ paraformaldehyde/ $4 \%$ sucrose/ $1 \times$

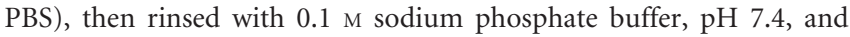
dissected away from skin and other tissue by hand using forceps. Dissected larval brains were further permeablized with $1 \mathrm{mg} / \mathrm{ml}$ collagenase for $30 \mathrm{~min}$, blocked in IB (0.1 M phosphate buffer/0.2\% BSA/0.5\% Triton-X/2\% normal goat serum) for $1 \mathrm{~h}$, then stained with diluted primary antibodies 1:50 anti-neurofilament (3A10, gift from T. Jessell) and 1:400 rabbit anti-GFP overnight at $4^{\circ} \mathrm{C}$, washed three times with $\mathrm{IB}$, and stained with 1:400 each Alexa488-goat-anti-rabbit and Alexa594-goat-anti-mouse. Stained samples were washed with phosphate buffer for $30 \mathrm{~min}$, transferred to Vectashield medium, then mounted, and imaged ventrally on a Zeiss LSM710 confocal microscope. As with younger larvae, tails were used to confirm the genotype of each larva following imaging.

Laser ablation of hindbrain interneurons. Targeted cell ablation was performed on using a MicroPoint Computer-Controlled ablation system (Andor Technology) consisting of a nitrogen-pumped dye laser (wavelength $435 \mathrm{~nm}$ ) controlled by Slidebook (version 5.0) on a spinning disc confocal microscope (Olympus). Ablation laser settings ranged from power $68-75$ depending on the age of the cumerin dye. The 3 dpf larvae were mounted in $1.2 \%$ low melt agarose for neural ablation, carrying 2 copies of the j1229a GFP enhancer trap transgene to visualize the Mauthner/MiD2/MiD3 array. As it was often not possible to clearly distinguish among labeled MiD2cm/MiD2i and among labeled MiD3cm/ $\mathrm{MiD} 3 \mathrm{cl} / \mathrm{MiD} 3 \mathrm{i}$, all 10 of these cell bodies were targeted for ablation in all larvae analyzed (see Fig. 5A). To aid in imaging, embryos were raised in $0.2 \mathrm{~mm}$ phenylthiourea from $24 \mathrm{hpf}$ through ablation, then transferred to E3 embryo media after ablation was confirmed. Neural ablation was verified $1-2 \mathrm{~h}$ after laser application, and only those individuals where all MiD2/MiD3 neurons were unambiguously ablated without disturbing the nearby Mauthner neurons were considered in the analysis (see Fig. $5 B$ ). Ablated and control larvae recovered from handling for $3 \mathrm{~d}$ in E3 embryo media before any behavioral analysis.

\section{Results}

\section{The spaced out phenotype is caused by mutations in the $d c c$} guidance receptor

We previously identified two zebrafish mutant spaced out alleles $\left(s p o^{t s 239}\right.$ and $\left.s p o^{t m 272 b}\right)$ based on a larval behavioral phenotype at 5-6 dpf (Granato et al., 1996). At this stage, wild-type siblings respond to startling stimuli with a high speed turn away from the stimulus followed by left/right alternating tail bends beginning on the opposing side (Kimmel et al., 1974). This behavioral response, the startle response, is highly stereotyped and easily elicited using tactile or acoustic stimuli (Liu and Fetcho, 1999; Burgess and Granato, 2007a). Although spaced out larvae respond readily to tactile or acoustic stimuli, they often do so with repeated bends to the same side, consistent with defects in the neural circuits governing the startle response (Granato et al., 1996). To identify the affected gene in spaced out individuals, we performed bulk segregant analysis on behaviorally identified mutant larvae using the $\operatorname{spo}^{t s 239}$ allele (Michelmore et al., 1991; Burgess et al., 2009). Consistent with prior mapping (Geisler et al., 2007), we found several polymorphic markers mapped to chromosome 5 with strong genetic linkage to spaced out, and placed the lesion between the genes TEK tyrosine kinase, endothelial (tek), and methyl-CpG binding domain protein 2 ( $m b d 2)$ (Fig. $1 A$ ). Subsequent recombinant mapping revealed two tightly linked length polymorphism markers, and BLAT alignments of these marker sequences placed them on genomic contigs downstream of the first exon of $d c c$ and upstream of the third exon of $d c c$, respectively (Fig. 1A) (Kent, 2002). Using published $d c c$ cDNA sequences as a guide (Fricke and Chien, 2005), we cloned and sequenced full-length $d c c$ cDNA from mutant larvae of both mutant spaced out alleles. In $s p o^{t s 239}$ mutants, this did not reveal any changes in the DCC coding sequence. In contrast, sequence analysis of $s p o^{t m 272 b}$ mutants revealed a $\mathrm{T} \rightarrow \mathrm{A}$ change in $d c c$, substituting the nonpolar isoleucine 790 with a positively charged asparagine (Fig. $1 B, C, \mathrm{I}^{790} \rightarrow \mathrm{N}$ ). The $\mathrm{I}^{790}$ residue falls within the fourth fibronectin Type III domain of DCC, located in a highly conserved $\beta$-strand region of the protein in vertebrates and invertebrates alike (Fig. 1C) (Bennett et al., 1997; Kruger et al., 2004). Importantly, the fourth fibronectin Type III domain of DCC has been implicated in the binding of its ligand Netrin, suggesting that the $\mathrm{I}^{790} \rightarrow \mathrm{N}$ mutation might disrupt DCCNetrin interaction and be causative for the spaced out behavioral phenotype.

We tested the impact of the $\mathrm{I}^{790} \rightarrow \mathrm{N}$ mutation on DCCNetrin interaction in mammalian cell culture using a Netrin overlay-binding assay. Full-length wild-type zebrafish $d c c$ and $d c c^{t m 272 b}$ were EGFP tagged and expressed in Cos-7 cells. Wildtype and mutant DCC-GFP were expressed at similar levels and both colocalized with a plasma membrane-targeted RFP marker (Fig. 1D,E; data not shown). FLAG epitope tagged Netrin-1 was incubated on transfected cells, and bound Netrin was detected by immunofluorescence (Fig. 1D,E). To measure background adherence of Netrin to cells, we similarly treated cells expressing the EGFP-tagged transmembrane Muscle-Specific Kinase (MuSK-GFP), which does not interact with Netrin (Fig. $1 F$ ). FLAG-Netrin was significantly enriched and colocalized with wild-type zebrafish DCC on the plasma membrane, compared with the MuSK-GFP control (Fig. $1 D, D^{\prime}, G ; p=4.5 \times 10^{-9}$ ). In contrast, no significant FLAG-Netrin enrichment over control was observed when mutant $\mathrm{DCC}\left(\mathrm{I}^{790} \rightarrow \mathrm{N}\right)$ was expressed at similar levels (Fig. $1 E, E^{\prime}, G ; p=0.69$ vs MuSK-GFP, $p=2.7 \times 10^{-10}$ vs DCC(WT)GFP). Thus, the $\mathrm{I}^{790} \rightarrow \mathrm{N}$ mutation of $s p o^{\text {tm272b }}$ compromises the ability of DCC to bind its ligand Netrin in vitro.

To determine whether the $\mathrm{I}^{790} \rightarrow \mathrm{N}$ missense mutation causes the spaced out behavioral defects, we first genotyped offspring from crosses between heterozygous $s p o^{t m 272 b} /+$ adults, and confirmed that the $\mathrm{I}^{790} \rightarrow \mathrm{N}$ mutation was present in $100 \%$ of larvae displaying the characteristic spaced out behavioral phenotype ( $n=192$ larvae). Second, we obtained fish strain carrying a viral insertion in the $5^{\prime}$ UTR of the DCC gene $\left(d c c^{z m 130198}\right.$, Fig. $\left.1 H\right)$ (Jao et al., 2008). Quantitative RT-PCR using $d c c^{z m 130198}$ homozygous embryos or $s p o^{t s 239}$ mutant larvae revealed a strong reduction in dcc mRNA in both of these mutants (Fig. $1 I ; p \leq$ $0.0001)$. Kinematic analysis of the acoustic startle response of homozygous $d c c^{z m 130198}$ larvae revealed a striking increase in turning angle magnitude, characteristic for larvae mutant for either spaced out allele, $s p o^{t s 239}$ and $s p o^{t m 272 b}$ (Fig. $2 A$, described in detail below). Furthermore, $s p o^{t m 272 b} / \mathrm{dcc}^{z m 130198}$ trans-heterozygous individuals also exhibited abnormal acoustic startle responses with the same characteristic exaggerated turn angles observed in spaced out mutants (Fig. $2 A$ ). Thus, the $d c c^{z m 130198}$ insertion allele fails to complement the spaced out mutation, confirming that the spaced out behavioral phenotype is due to an $\mathrm{I}^{790} \rightarrow \mathrm{N}$ missense mutation in the $d c c$ gene. We will refer to spaced out as $d c c$ hereafter.

\section{Startle response performance and rhythmic swimming are disrupted in $d c c$ mutants}

We next wanted to determine whether the behavioral deficits in left/right body coordination observed in $d c c$ mutants are caused by the loss of commissural neuronal connectivity or by aberrant 
A

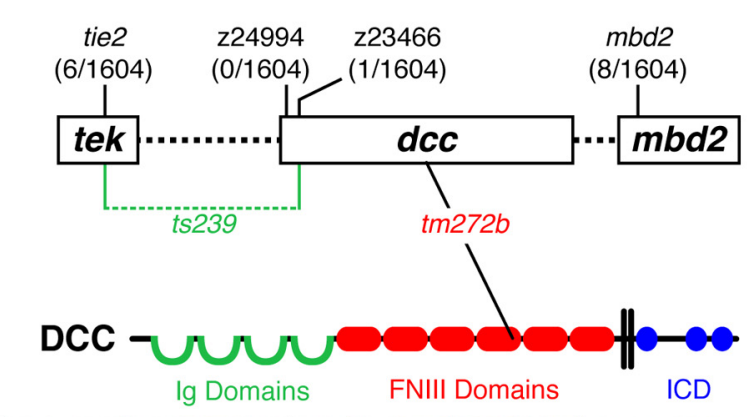

B

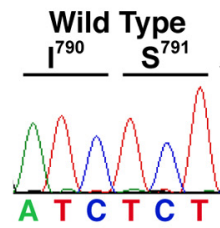

C D. melanogaster

C. elegans

M. musculus

H. sapiens

D. rerio (Wild Type)

D. rerio $\left(d c c^{t m 272 b}\right)$

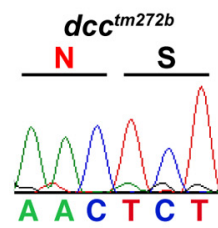

YVVS LRAR

YV I S LRAF

YV I S LKAF

YV I S LKAF

YV I S LKAF

YVNS LKAF
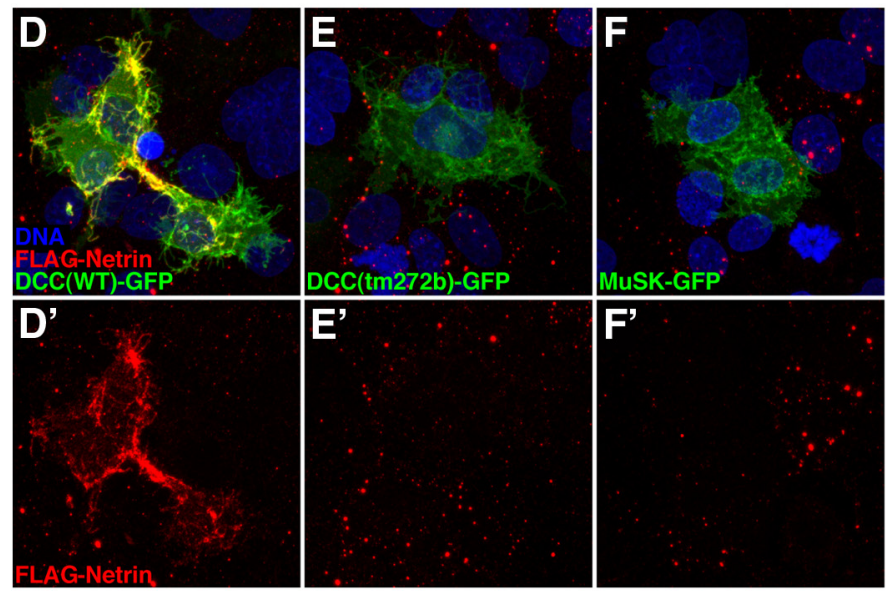

G

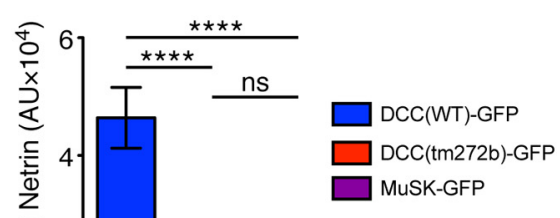

H

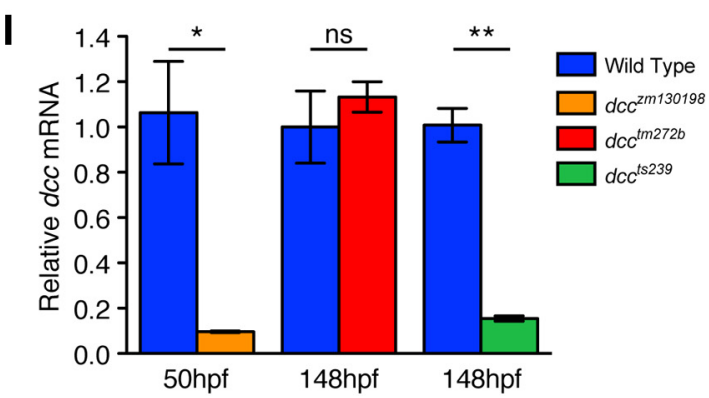

Figure 1. The zebrafish dcc gene is mutated in spaced out. A, Recombination mapping placed spo ${ }^{\text {ts239 }}$ between a SNP marker in the tek gene (tie2, 6 of 1604 meioses) and an SSLP marker in the presumptive second intron of $d c c$ (z23466, 1 of 1604 meioses). Although no mutations were observed in the $d c c$ coding sequence of $s p 0^{t s 239}$, a point mutation was detected in the spo ${ }^{\text {tm } 272 b}$ allele in the fourth Fibronectin Type III domain of DCC (FNIII, red ovals). The immunoglobulin-like domains (green horseshoes), transmembrane domain (black vertical bars), and cytoplasmic domains (conserved P1, P2, and P3 domains in blue) are all unperturbed in these alleles. $\boldsymbol{B}$, The spo ${ }^{t m 272 b}$ allele carries a T-to-A mutation, producing an isoleucine-to-asparagine missense in the DCC protein sequence ( ${ }^{790} \rightarrow \mathrm{N}$ ). $\boldsymbol{C}$, The isoleucine residue disrupted in the spo ${ }^{\text {tm272b }}$ allele $\left({ }^{790}\right)$ is in a highly conserved region of the fourth fibronectin domain of DCC. D-F, Binding of FLAG-Netrin to Cos-7 cells transfected with wild-type zebrafish dcc-egfp $(\boldsymbol{D})$, dcctm272b -egfp $(\boldsymbol{E})$, or musk-egfp $(\boldsymbol{F})$. FLAG-Netrin signal alone for each is shown in $\boldsymbol{D}^{\prime}-\boldsymbol{F}^{\prime}$. $\mathbf{G}$, Quantification of background-corrected total cell immunofluorescence of the FLAG epitope of GFP-positive transfected cells following FLAG-Netrin overlay. The number of cells analyzed per condition are shown at the base of each column. ${ }^{* * *} p<0.0001,{ }^{*}$ ns, not significant. $\boldsymbol{H}$, The $d c^{2 m 130198}$ allele carries a $5.2 \mathrm{~kb}$ retrotransposon insertion in the $5^{\prime}$ UTR of $d c c, 106$ nucleotides upstream of the start codon. I, Quantitative RT-PCR showed a significant decrease in $d c c$ transcript levels in $d c c^{2 m} 130198$ homozygotes at $50 \mathrm{hpf}\left({ }^{*} p=0.0342\right)$ and in $d c c^{t 5239}$ homozygotes at $148 \mathrm{hpf}\left({ }^{* *} p=0.0015\right)$. ns, not significant. Four independent RNA samples were analyzed in each condition.

ipsilateral connections. For this, we turned to the startle response circuit because $d c c$ mutants display overt startle response defects, and because the hindbrain neural circuits underlying the startle response consist of a small number of well-characterized neurons (Granato et al., 1996; Bhatt et al., 2007; Koyama et al., 2011). We first characterized startle defects of $d c c$ mutants, then identified which commissural connections of the startle response circuitry depend on $d c c$ function, and finally determined whether the behavioral startle phenotype observed in $d c c$ mutants was caused by the loss of commissural neuronal connectivity, or by aberrant ipsilateral connections.

The larval zebrafish startle response, triggered by tactile or acoustic stimuli, can be broken down into a stereotypic series of discrete and quantifiable movement patterns (Burgess and
Granato, 2008; Fero et al., 2011; McClenahan et al., 2012). Milliseconds after the stimulus, larvae initiate a high-speed turn (Cbend, "B1") directed away from the perceived stimulus (Fig. 2B) (Kimmel et al., 1974; Burgess and Granato, 2007a). This C-bend turn is followed by a weaker turn in the opposite direction (Counterbend, "B2"), and then a bout of rhythmic left/right swimming undulations ("B3, B4" etc; Fig. 2C). The net result is that larvae move rapidly away from potentially threatening stimuli. Throughout the entire movement sequence, the body axis bends strictly alternate between the rightward and leftward directions, and the counterbend ("B2") represents the initiation of this alternation. Thus, left/right coordination is critical throughout the entire startle response and can be quantified at millisecond resolution, as shown in Figure 2C, where approximate total body 

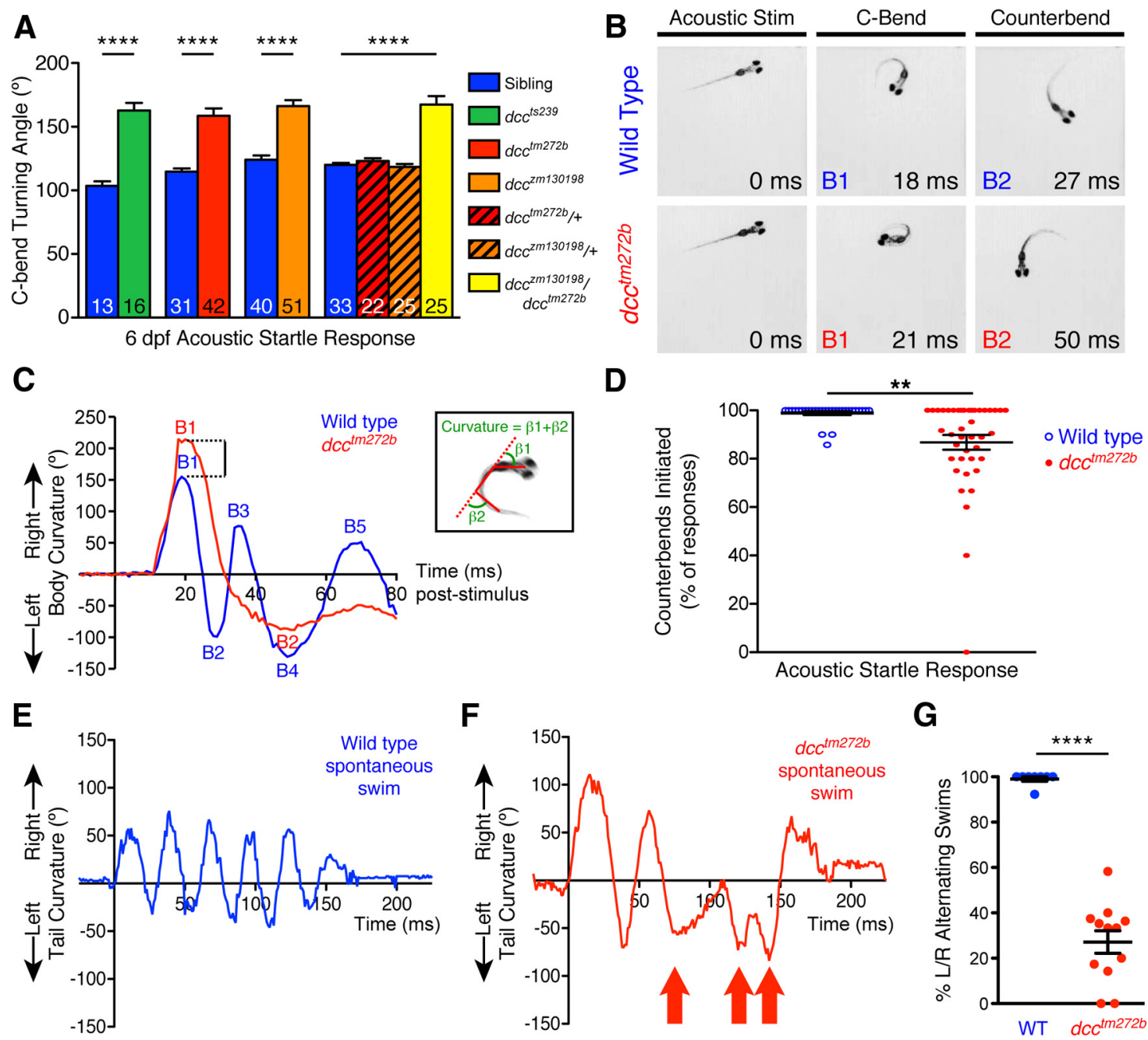

Figure 2. The acoustic startle responses of $d c c$ mutant larvae display exaggerated magnitude and disrupted counterbends. A, Homozygous $d c^{t 5239}, d c c^{t m 272 b}$, and $d c c^{2 m 130198}$ larvae show a significant increase in maximum head turning angle during short latency acoustic startle responses compared with their siblings. ${ }^{* * * *}<0.0001$ for each pair. Trans-heterozygous $d c^{t m 272 b}$ /

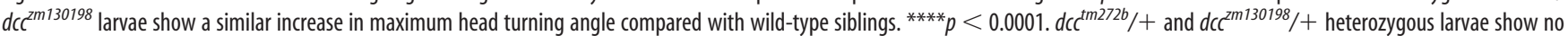
significant acoustic startle defects. Larvae received 20 acoustic stimuli each, and numbers of larvae analyzed per genotype are shown at the base of each column. $\boldsymbol{B}, \boldsymbol{C}$, Representative time series of $6 \mathrm{dpf}$ wild-type ( $\boldsymbol{B}$, top) and $d c^{t m 272 b}$ mutant ( $\boldsymbol{B}$, bottom) zebrafish larvae responding to acoustic stimuli. $\boldsymbol{B}$, Panels represent the points of maximal body curvature for the $(-$ bend ("B1") and counterbend ("B2") of the acoustic startle response. The total body curvatures of the larval responses in B are graphed in $C>80 \mathrm{~ms}$ after initiation of the acoustic stimulus. $C$, Black bracket represents the increase in maximal body curvature achieved during the C-bend of $d c c$ mutants. D. The average frequency of counterbends performed following acoustically evoked C-bends by $6 \mathrm{dpf} d c c^{t m 272 b}$ mutant larvae ( $d c c, n=42$ in red) and their wild-type siblings (WT, $n=31$ in blue) across 20 identical acoustic stimuli. ${ }^{* *} p=0.0054$ (one-tailed $t$ test with Welch's correction for unequal variances). Each point graphed represents a single larva. $\boldsymbol{E}, \boldsymbol{F}$, Tail curvature during a spontaneous swim maneuver performed by a $6 \mathrm{dpf}$ wild-type sibling $(\boldsymbol{E})$ and a dctm272b mutant larva $(\boldsymbol{F})$. Three repeated leftward tail bends by the mutant are highlighted with arrows in $\boldsymbol{F}$. $\boldsymbol{G}$, Frequency of spontaneous swim maneuvers with strictly left/right alternating tail bends for wild-type ( $n=8$ larvae) and $d c c^{t m 272 b}$ mutants $\left(n=12\right.$ larvae). ${ }^{* * *} p<0.0001$. Each point represents a single larva.

curvature is graphed as a function of time with rightward and leftward curvature represented as positive and negative values, respectively.

To examine the precise movement deficits in $d c c$ mutants, we examined their performance in response to acoustic startle stimuli at millisecond resolution (Fig. $2 A-D$ ). Following acoustic stimuli, $d c c$ mutants displayed latencies and response frequencies similar to those observed in wild-type siblings (Fig. 2C). In contrast, $d c c$ mutants exhibited specific defects in both movement magnitude and left/right alternation throughout the entire startle response. Specifically, the initial C-bends were exaggerated compared with their wild-type siblings, with mutants often contacting the tip of their tail with their heads (Fig. $2 A-C$ ). Similarly, $d c c$ mutant $\mathrm{C}$-bend duration, head turning angle, and maximal body curvature were all significantly larger than those measured in wild-type siblings (Fig. $2 A-C$; note increase in B1 body curvature peak in $C)$. No significant defects were observed in acoustic startle performance of heterozygous $d c c^{t m 272 b} /+$ or $d c c^{z m 130198} /+$ larvae (Fig. 2A; data not shown).

To determine whether rhythmic left/right alternation was disrupted in additional behavioral contexts, we examined spontaneous swim bouts of wild-type and $d c c^{\operatorname{tm} 272 b}$ larvae (Fig. 2E-G). Spontaneous movements of $d c c^{t m 272 b}$ larvae were more kinematically variable than those of their wild-type siblings, and all mutants displayed a reduced frequency of spontaneous swim bouts with strictly left/right tail bend alternation (Fig. $2 F, G ; p<$ $0.0001)$. Together, these data reveal specific defects throughout the entire sequence of the left/right alternating spontaneous swimming and acoustic startle response behaviors of $d c c$ larvae, indicating that $d c c$ is required for the appropriate assembly and function of the neuronal circuits controlling multiple movement patterns of during rhythmic swimming and the startle response. 
A

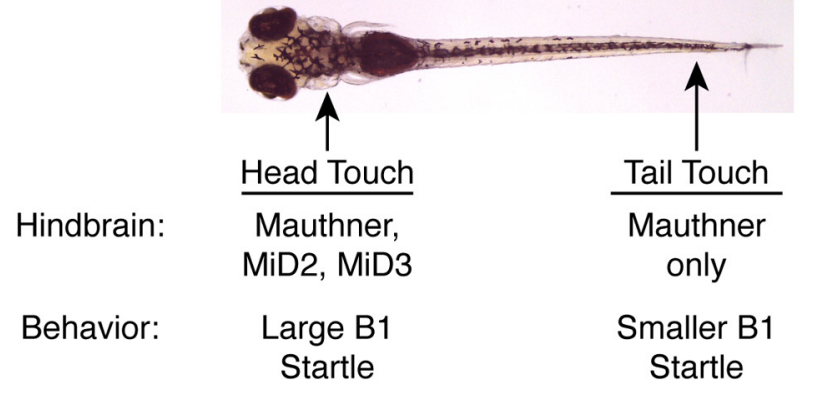

Counterbend
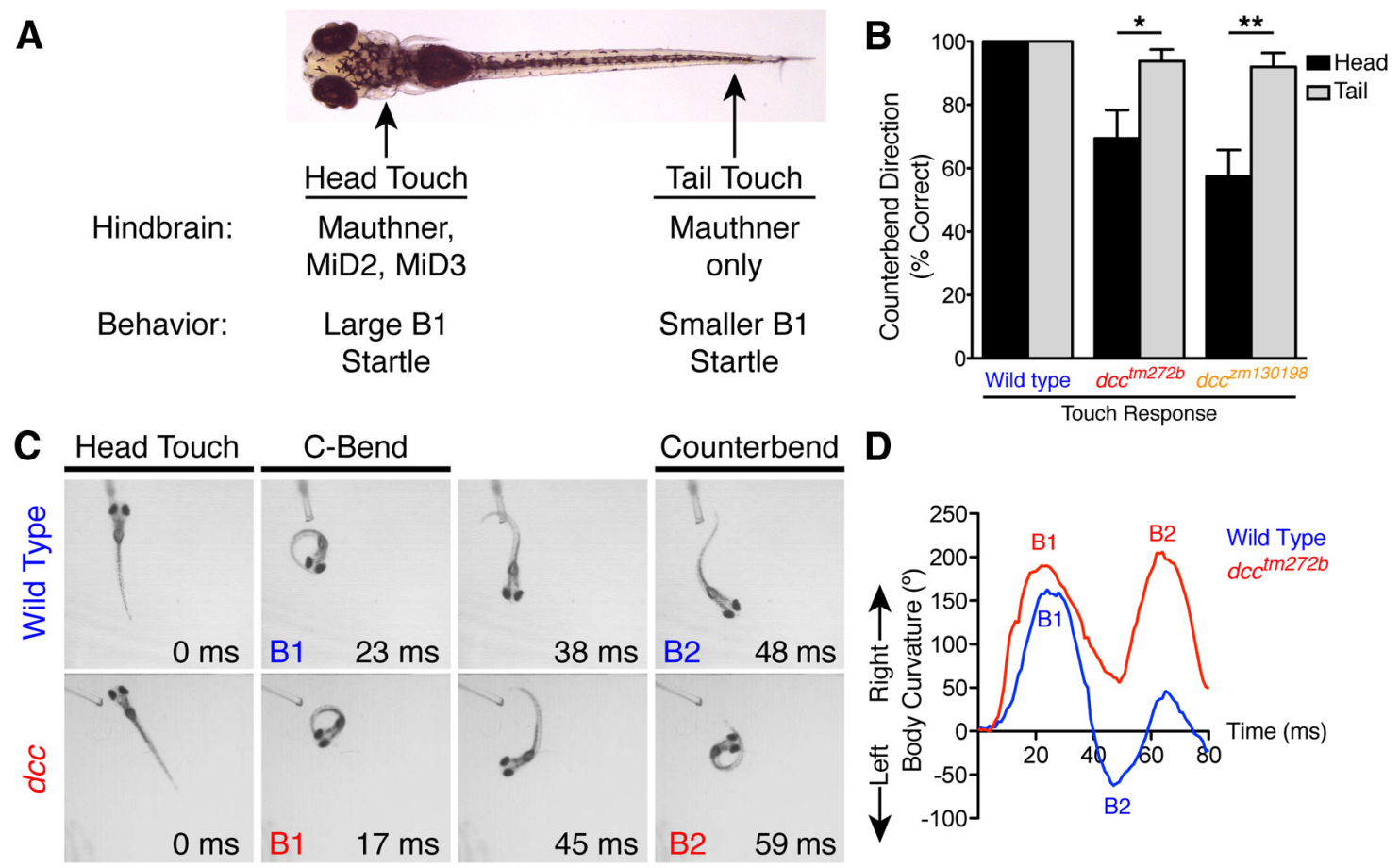

\section{。}
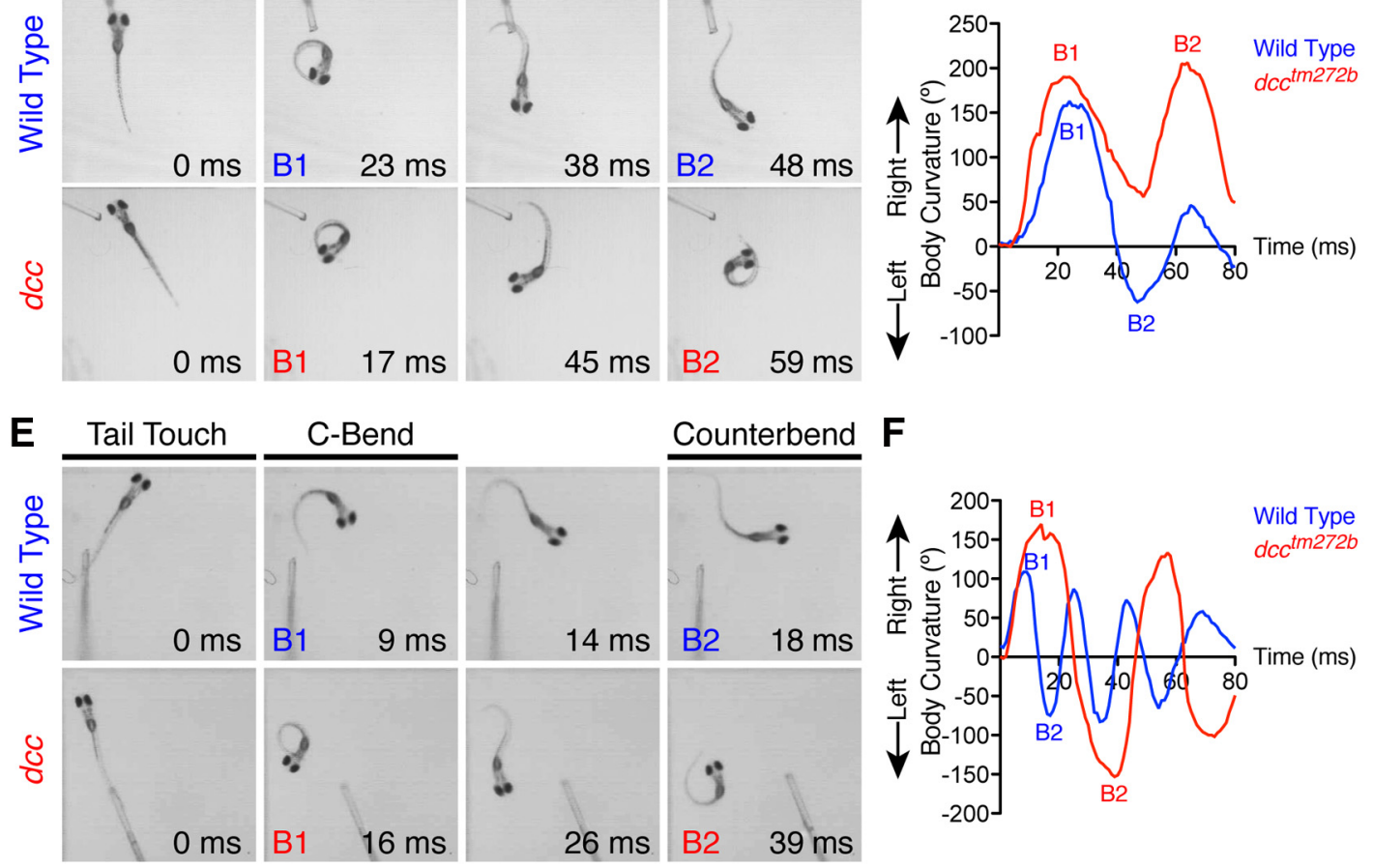

Figure 3. dec is required for counterbend directionality during touch-evoked startle responses. $\boldsymbol{A}$, Schematic comparison of known neuronal and behavioral differences between head and tail touch-evoked startle responses (Liu and Fetcho, 1999; Gahtan et al., 2002; Bhatt et al., 2007; Kohashi and Oda, 2008). B, Average frequency of touch-evoked counterbends correctly directed to the opposite side from the initial C-bend. Each larva was tested at $6-8 \mathrm{dpf}$ with $10-15$ tactile stimuli to the head and tail ( $n=5$ wild-type larvae, $12 \mathrm{dcc} \mathrm{c}^{\mathrm{tm} 272 b}$ larvae, $\left.7 \mathrm{dc} \mathrm{c}^{2 \mathrm{~m} 130198} \mathrm{larvae}\right){ }^{*} p=0.0244$ (two-tailed pairwise $t$ test). ${ }^{* *} p=0.0025$ (two-tailed pairwise $t$ test). $C-F$, Representative time series of 6 dpf wild-type $\left(\boldsymbol{C}, \boldsymbol{E}\right.$, top) and $d c c^{t m 272 b}$ mutant $(\boldsymbol{C}, \boldsymbol{E}$, bottom) larvae responding to tactile stimuli to the head $(\boldsymbol{C}, \boldsymbol{D})$ or tail $(\boldsymbol{E}, \boldsymbol{F}) . \boldsymbol{C}, \boldsymbol{E}$, Panels include the points of maximal body curvature for the (-bend ("B1") and counterbend ("B2") of the startle responses. $\boldsymbol{D}, \boldsymbol{F}$, The total body curvatures of the larval responses depicted in $\boldsymbol{C}$ and $\boldsymbol{E}$ are graphed over $80 \mathrm{~ms}$ following initiation of the startle maneuver, with the wild-type response in blue and the $d c^{\mathrm{tm} 272 b}$ mutant response in red.

$d c c$ controls counterbend initiation and directionality

Given the well-established role of hindbrain neurons in the startle response, we examined whether $d c c$ regulates counterbend performance through hindbrain interneurons during the startle response. Following acoustic stimuli, wild-type larvae perform a rapid C-bend immediately followed by a counterbend turn to the alternate side (Fig. $2 B ; n=31$ larvae). In contrast, most $d c c$ mutant larvae displayed a significantly reduced acoustically evoked counterbend frequency, consistent with defects in counterbend initiation (Fig. $2 D ; n=23 / 41 d c c^{t m 272 b}$ larvae, $p<0.001$ ). In those cases when $d c c$ mutants performed counterbends, some were directed to the same side as the C-bend, further confirming defects in the initiation of left/right alternation $\left(n=7 / 40 d c c^{t m 272 b}\right.$ larvae).

In addition to acoustically evoked startle responses, we examined tactile-evoked startle responses, as the latter allows us to differentiate between subsets of hindbrain neurons executing the behavior, depending on whether tactile stimuli are delivered to the head or the tail (Fig. 3A) (Liu and Fetcho, 1999). Both tactile stimuli recruit the hindbrain Mauthner command neurons and appear to activate the same sets of spinal interneurons (Bhatt et al., 2007). However, head touch-evoked startle responses additionally recruit the reticulospinal Mauthner homologs $(\mathrm{MiD} 2 \mathrm{~cm}$, $\mathrm{MiD} 3 \mathrm{~cm}$, and $\mathrm{MiD} 3 \mathrm{cl}$ ), whereas tail touch-induced responses do not require $\mathrm{MiD} 2 \mathrm{~cm}$ and MiD3cm (O'Malley et al., 1996; Liu and Fetcho, 1999; Gahtan et al., 2002; Kohashi and Oda, 2008). Compared with acoustic stimuli, we observed even more striking defects in counterbend direction when startle responses were evoked by tactile stimuli. Whereas wild-type sibling larvae always performed counterbends in the correct direction when touched, opposite to the initial C-bend, most $d c c$ mutants performed some touch-evoked counterbends to the same side as the initial C-bend (Fig. $3 B-D ; n=9 / 12 d c c^{\text {tm272b }}$ larvae, $n=7 / 7 d c c^{z m 130198}$ larvae). Intriguingly, the defect in counterbend direction was significantly more pronounced when $d c c$ larvae were touched on the head than when the same larvae were touched on side of their tail (Fig. $3 B-F ; n=12 d c c^{t m 272 b}$ larvae, $n=7 d c c^{z m 130198}$ larvae, 

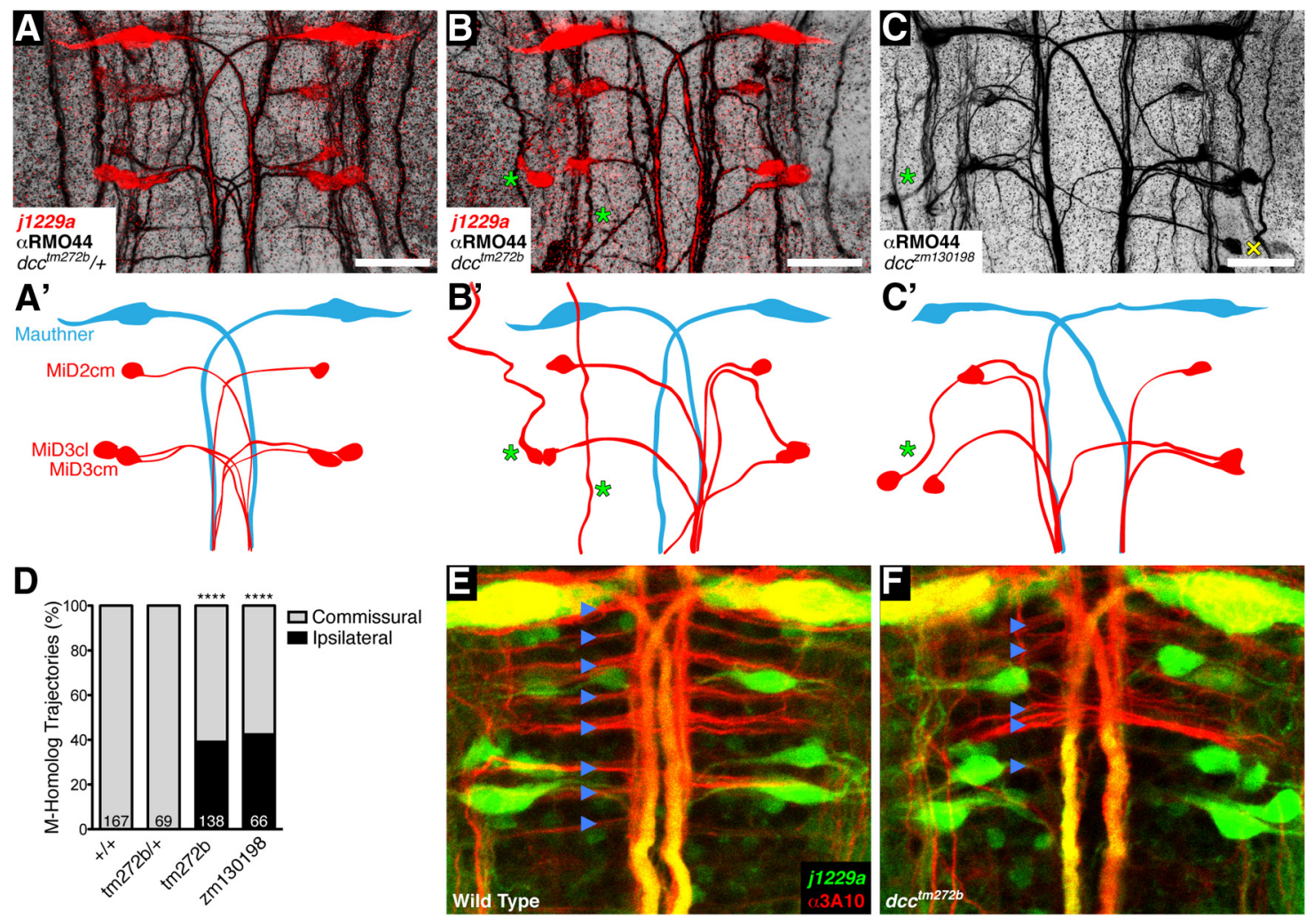

Figure 4. dec is required for commissural axonal projections of hindbrain interneurons, including the Mauthner/MiD2/MiD3 array. A-C, Confocal projections of hindbrain rhombomeres $4-6$ of

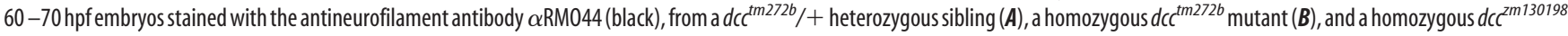
mutant (C). $A, B$, The GFP enhancer trap transgene j1229a was also present to colabel the Mauthner array cell bodies with anti-GFP (red). Green asterisks indicate MiD3cl axons aberrantly extending laterally and/or rostrally. Yellow " $X$ " indicates the cell body of an unscored T-reticular neuron extending a commissural axon through rhombomere 6 in panel $C$. White scale bars, $36 \mu \mathrm{m}$. For clarity, camera lucida tracings of the Mauthner arrays in these projections are presented in $A^{\prime}-C^{\prime}$. Mauthner axons are in blue (rhombomere 4 ) and Mauthner homolog axons are in red (MiD2 $\mathrm{cm}$ pair from rhombomere 5, MiD3cl and MiD3cm pairs from rhombomere 6). The MiD3cl axon in (extends rostrally out of the presented image, then turns and extends ipsilaterally toward the posterior in a more lateral axon tract. $\boldsymbol{D}$, Quantification of commissural versus ipsilateral axonal projections of hindbrain M-homolog neurons $(\mathrm{MiD} 2 \mathrm{~cm}, \mathrm{MiD} 3 \mathrm{~cm}, \mathrm{MiD} 3 \mathrm{cl})$ stained by $\alpha \mathrm{RM} 044$ for wild-type $(+/+, n=$ 33 embryos), heterozygous ( $d c^{t m 272 b} /+, n=14$ embryos), and $d c c$ mutants ( $d c c^{t m 272 b}$ and $d c c^{2 m 130198}, n=24$ and 13 embryos, respectively). The number of scored neurons is listed at the base of each bar. ${ }^{* * *} p<0.0001 . E, F$, Confocal projections of hindbrain rhombomeres $4-7$ of 6 dpf larval brains stained with an anti-neurofilament antibody ( $\alpha 3 A 10$, red) and $\alpha$ GFP (green), from sibling $(\boldsymbol{E})$ and $d c^{t m 272 b}$ mutants $(\boldsymbol{F})$ carrying 2 copies of the j1229a GFP enhancer trap transgene. Blue arrowheads indicate discrete hindbrain commissure bundles labeled by $\alpha 3 \mathrm{~A} 10$.

$p=0.0244$ and $p=0.0025$, respectively, two-tailed paired $t$ test). This behavioral difference strongly suggests that $d c c$ regulates a key population of neurons recruited in response to head touches that is not used during escapes evoked by tail touch or acoustic stimuli, consistent with the idea that $d c c$ is required for $\mathrm{MiD} 2 \mathrm{~cm} /$ $\mathrm{MiD} 3 \mathrm{~cm} / \mathrm{MiD} 3 \mathrm{cl}$ development and/or function. Overall, our behavioral data reveal a key role for $d c c$ in regulating the performance and directionality of counterbends in the context of the startle responses and strongly suggest a role for $d c c$-dependent hindbrain circuits in regulating counterbends.

$d c c$ mutants exhibit defects in the commissural trajectories of identified hindbrain neurons

Given the pronounced counterbend direction defect of $d c c$ larvae when touched on the head, we hypothesized that $d c c$ regulates the axonal projections of the $\mathrm{MiD} 2 \mathrm{~cm} / \mathrm{MiD} 3 \mathrm{~cm} / \mathrm{MiD} 3 \mathrm{cl}$ reticulospinal Mauthner homologs. To test this, we examined the commissural trajectories of the Mauthner hindbrain array consisting of a bilateral pair of Mauthner neurons (Fig. $4 A, A^{\prime}$, blue) and their segmental homologs, MiD2cm, MiD3cm, and MiD3cl (Fig. $4 A, A^{\prime}$, red). In wild-type siblings, the $j 1229 a$ GFP enhancer trap transgene labels the cell bodies of the Mauthner neurons and their homologs, and the commissural axons of the Mauthner/ MiD2 $\mathrm{cm} / \mathrm{MiD} 3 \mathrm{~cm} / \mathrm{MiD} 31$ pairs can be identified by neurofila- ment antibody staining at $60 \mathrm{hpf}$ (181 of 181 commissural MiD2cm/MiD3cm/MiD3l axons, $n=33$ wild-type embryos) (Waskiewicz et al., 2001). Whereas commissural axons from both Mauthner neurons were generally observed in $d c c$ mutants, $1-5$ $\mathrm{MiD} 2 \mathrm{~cm} / \mathrm{MiD} 3 \mathrm{~cm} / \mathrm{MiD} 3 \mathrm{cl}$ axons failed to project contralaterally in $100 \%$ of $d c c^{t m 272 b}$ and $d c c^{z m 130198}$ embryos examined (Fig. $4 B-D ; d c c^{\text {tm } 272 b}: 54$ of 138 misprojecting axons, $n=24$ larvae; $d c c^{z m 130198}: 28$ of 66 misprojecting axons, $n=13$ larvae, $p<$ 0.0001 vs wild-type siblings for each, 1-tailed Fisher Exact test). Cell bodies of these neurons, particularly MiD3cl, sometimes appeared to be more laterally positioned relative to axon tracts (Fig. $4 C$, left MiD3cl); and in some cases, labeled axons projected laterally and/or rostrally in novel ectopic paths to join ipsilateral axon tracts, a phenotype never observed in wild-type (Fig. $4 B, C$, green asterisks).

In addition, we examined commissural axons of the more rostral RoL2 reticulospinal neurons, which are also detectable at this stage by $\alpha$ RMO44 staining ( $2.0 \pm 0.0$ commissural axons/ embryo, $n=30$ wild-type embryos) (Metcalfe et al., 1986; Hatta, 1992). In most $d c c$ mutants, one or both of these RoL2 commissural axons were absent (15 of $24 d c c^{\text {tm272b }}$ mutants with RoL2 defects, 6 of $13 d c c^{z m 130198}$ mutants with RoL2 defects; $p<0.001$ for each, one-tailed Fisher's exact test; data not shown), suggesting that $d c c$ is required broadly to regulate axonal guidance of 
commissural hindbrain neurons. Therefore, we examined the later-developing extensive scaffold of commissural hindbrain axonal tracts in $d c c$ mutant larvae, using a neurofilament antibody and the $j 1229 a$ enhancer trap line to provide spatial landmarks (Burgess et al., 2009). Specifically, we focused on the regular ladder-like array of commissural interneuron axons in rhombomeres $4-7$ of the caudal larval hindbrain. At 6 dpf, wild-type larvae reliably had 8 commissural bundles, whereas $d c c$ mutants showed disorder in these commissures, with variable reductions in the number of distinguishable commissural tracts (Fig. 4E, F; $n=6$ wild-type and $6 d c c^{t m 272 b}$ ). Thus, $d c c$ regulates commissural guidance of multiple hindbrain neurons, in particular the $\mathrm{MiD} 2 \mathrm{~cm}, \mathrm{MiD} 2 \mathrm{~cm}$, and $\mathrm{MiD} 3 \mathrm{cl}$ neurons implicated in the head touch-evoked startle response.

\section{Counterbend directionality defects in $d c c$ mutants are caused by ipsilateral MiD2 $\mathrm{cm} / \mathrm{MiD} 3 \mathrm{~cm} / \mathrm{MiD} 3 \mathrm{cl}$ projection}

Finally, we wanted to determine whether the defects in counterbend directionality in $d c c$ mutants are caused by the loss of $\mathrm{MiD} 2 \mathrm{~cm} / \mathrm{MiD} 3 \mathrm{~cm} / \mathrm{MiD} 3 \mathrm{cl}$ commissural connectivity, or by aberrant ipsilateral connections formed by these neurons. Based on current models, there are two attractive explanations for the observed behavioral defects. First, in wild-type larvae, MiD2cm/ $\mathrm{MiD} 3 \mathrm{~cm} / \mathrm{MiD} 3 \mathrm{cl}$ neurons make synaptic connections in the contralateral spinal cord critical to specify the counterbend directionality. In the $d c c$ mutants, these contralateral projections might be reduced or absent, thereby impairing counterbend directionality. To test this first possibility, we laser ablated MiD2/ MiD3 homologs in wild-type larvae and then examined the fidelity of their counterbend direction following head touch stimuli (Fig. $5 A, B$ ). Ablation of the MiD2/MiD3 homologs in wild-type larvae resulted in head-touch responses indistinguishable from unablated control individuals, with $100 \%$ of responses performing strict left/right alternation of the C-bend and counterbend (Fig. $5 C ; n=5$ larvae). Thus, the MiD2/MiD3 homologs are not required to specify counterbend direction.

A second possibility is that in $d c c$ mutants some of the MiD2/ MiD3 homologs fail to project contralaterally, and instead project ipsilaterally down the spinal cord where they form ectopic synaptic connections with ipsilateral interneurons and/or spinal motor neurons in addition to some appropriate contralateral synaptic connections. To test this second possibility, we ablated the MiD2/MiD3 homologs in $d c c$ mutant individuals and measured counterbend directionality. Following head touch stimuli, nonablated $d c c$ mutants performed counterbends that were frequently misdirected (Fig. 5C; $41 \pm 7.6 \%$ misdirected, $n=15$ larvae). In contrast, $d c c$ mutants in which the MiD2/MiD3 homologs had been ablated displayed a significant rescue of counterbend direction following head touch (Fig. $5 C$; $9.75 \pm 6 \%$ misdirected, $p=0.010$ for two-tailed $t$ test vs unablated $d c c$ mutants, $n=4$ larvae). Thus, $d c c$ is critical to govern the relative directionality of counterbends through its control of the commissural guidance of the Mauthner homologs, preventing inappropriate ipsilateral synaptic contacts. This demonstrates a functional role for DCC in regulating left/right alternation circuits in the hindbrain.

\section{Discussion}

In congenital MMD patients carrying causative mutations in the $D C C$ guidance receptor, a proposed cause for the movement deficiencies is inappropriate descending bilateral corticospinal tract projections. However, the direct impact of inappropriate corticospinal projections on motor behaviors in DCC mutant mice has been inconclusive (Dottori et al., 1998; Coonan et al., 2001;
Kullander et al., 2003; Fawcett et al., 2007). Here we address the role of ectopic ipsilateral descending projections in $d c c$ mutant zebrafish to determine the role of $d c c$ in regulating reticulospinal circuitry underlying descending motor control. Through targeted ablation of a small number of descending hindbrain interneurons, we demonstrate that inappropriate bilateral connections of $d c c$ mutant reticulospinal tracts are sufficient to evoke involuntary mirror movement-like behaviors.

\section{The spaced out behavioral phenotype is caused by a missense mutation in the $d c c$ guidance receptor}

We identified three independent mutant alleles that give rise to the zebrafish spaced out/dcc phenotype: $d c^{z m 130198}, d c c^{\text {tm239 }}$, and $d c c^{t m 272 b}$, respectively. In $d c^{z m 130198}$ (caused by a viral insertion) and $d c^{t m 239}$ homozygotes (caused by a presumptive promoter mutation; Fig. 1I), dcc mRNA levels are $>90 \%$ reduced, whereas $d c c^{t m 272 b}$ mutants carry a single $\mathrm{I}^{790} \rightarrow \mathrm{N}$ amino acid substitution in the fourth fibronectin Type III domain. Cell culture and in vitro pull-down experiments have previously implicated DCC's fourth and/or fifth fibronectin Type III domains as the Netrin binding site (Bennett et al., 1997; Geisbrecht, 2003; Kruger et al., 2004), and consistent with this we find that the $\mathrm{I}^{790} \rightarrow \mathrm{N}$ mutation significantly compromises DCC-Netrin interaction in cell culture (Fig. $1 D-G$ ), although we cannot exclude additional protein trafficking defects in zebrafish. Importantly, the strength of the behavioral phenotype observed in $d c c^{t m 272 b} \mathrm{mu}-$ tants is indistinguishable from that of $d c c^{t m 272 b} / d c c^{z m 130198}$ transheterozygotes (Fig. 2A). Furthermore, the axonal defects in the hindbrains of $d c^{t m 272 b}$ and $d c c^{z m 130198}$ mutants are $100 \%$ penetrant and observed at similar neuronal frequencies (Fig. 4D), providing compelling evidence that the spaced out phenotype is caused by loss-of-function mutations in the $d c c$ gene. Finally, heterozygous DCC patients present mirror movement phenotypes, whereas we only observe neural or behavioral phenotypes in homozygous $d c c^{t m 272 b}$ or $d c c^{z m 130198}$ zebrafish mutants (Figs. $2 A$ and $4 D$ ), indicating that these are hypomorphic alleles or that the simpler zebrafish Mauthner array is less sensitive to DCC levels than the human motor control circuits.

The spaced out/dcc mutation was initially identified as one of three mutants in which following startling stimuli, rather than performing alternating right and left bends, larvae perform multiple tail bends to the same side (Granato et al., 1996). The other two mutants are caused by mutations in the $R b 1$ tumor suppressor gene (space cadet) and in the robo3 guidance receptor (twitch twice) (Burgess et al., 2009; Gyda et al., 2012). In humans, robo3 mutations cause Horizontal Gaze Palsy with Progressive Scoliosis, disrupting left/right coordination of eye movements (Jen et al., 2004). Behavioral and neuroanatomical analyses of these three mutants have shown partially overlapping functions for these genes in regulating startle movements. For example, in $d c c$ and twitch twice/robo3 mutants, midline crossing of the Mauthner/MiD2/MiD3 array is affected, whereas these axonal processes are unaffected in space cadet/Rb1 mutants (Fig. 4). In contrast, in all three mutants the caudal array of hindbrain commissures is disorganized, suggesting that these functionally uncharacterized commissures regulate aspects of left/right movement coordination (Fig. 4) (Lorent et al., 2001; Burgess et al., 2009). Thus, like mutations in their human orthologs, mutations in the zebrafish robo3 and $d c c$ genes result in movement defects, consistent with the idea that the behavioral deficits are caused by disrupting evolutionarily well-conserved circuits. 

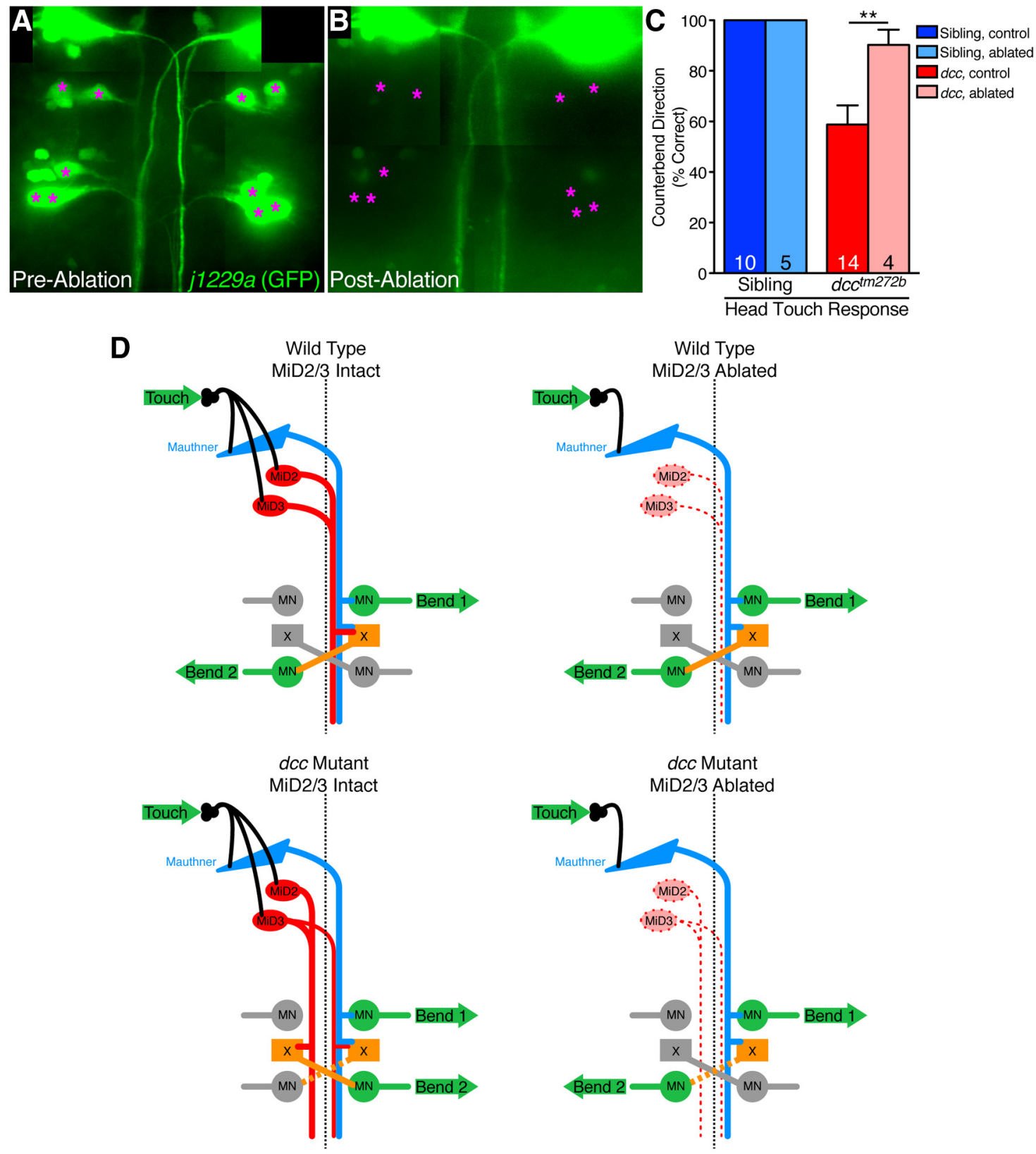

Figure 5. Ipsilaterally misprojecting MiD2/MiD3 neurons in dec mutants result in counterbend directionality defects. $A, B$, Confocal projections of hindbrain rhombomeres $4-7$ in a live 3 -d-old larva carrying 2 copies of the j1229a GFP enhancer trap transgene in green, immediately before laser ablation of the MiD2/MiD3 homologs $(\boldsymbol{A})$ and $1 \mathrm{~h}$ after ablation $(\boldsymbol{B})$. The cell body positions of Mauthner homologs in rhombomeres 5 and 6 targeted for ablation are marked with magenta asterisks. Images are composites of multiple overlapping z-projections, registered using Mauthner axons and unablated cells. Projections were individually adjusted for brightness and contrast to permit consistent visibility of the Mauthner array and debris, and to confirm ablation of neurons rather than photobleaching. C, Wild-type siblings with bilateral MiD2/MiD3 ablation (light blue) and unablated controls (dark blue), as well as similarly ablated and unablated dcc mutants (pink and red, respectively) were tested at $6 \mathrm{dpf}$ with 10 tactile stimuli to the head, and responses were specifically scored for counterbend directionality relative to the initial startle bend. Numbers of larvae analyzed per genotype are shown at the base of each column. All individuals carried 2 copies of the $j 1229 a$ GFP transgene to visualize the Mauthner/MiD2/MiD3 array for ablation. ${ }^{* *} p=0.0100$. D, A model for the role of MiD2/MiD3 neurons in the dcc mutant counterbend phenotype. Head touch activates the Mauthner/MiD2/MiD3 hindbrain array through the trigeminal sensory neurons (black). This reticulospinal array activates trunk motor neurons ("MN" in green) to initiate the contralateral (-bend ("Bend 1"), as well as proposed commissural interneurons of the caudal hindbrain and/or spinal cord (" $\mathrm{X}$ " in orange), which directly or indirectly activate motor neurons on the opposite side for the subsequent counterbend ("Bend 2"). In wild-type larvae where the MiD2/MiD3 neurons have been ablated (top right panel, red dotted lines indicating ablated neurons), Mauthner activity alone is sufficient to activate trunk motor neurons for the contralateral C-bend and commissural " $X$ " interneurons to allow an appropriate counterbend. In dec mutants (bottom left), these commissural interneurons are bilaterally activated, which resolves in some responses to produce a counterbend on the same side as the (-bend. In dec mutants where the MiD2/MiD3 neurons have been ablated (bottom right), this bilateral conflict is removed and appropriate counterbend direction is restored. Inactive neurons in each scenario are shaded gray.

\section{Ectopic ipsilateral descending projections in $d c c$ mutants produce mirror-like movements}

Bilaterally ablating wild-type larval MiD2/MiD3 neurons does not affect counterbend performance, whereas ablating these neurons in $d c c$ mutants restores counterbend performance. Com- bined, these results suggest a model in which $d c c$ disruption results in a mix of normal commissural and inappropriate ipsilateral MiD2/MiD3 axonal projections (Fig. 5D). In wild-type larvae, left-sided head stimuli activate the contralaterally projecting Mauthner, $\mathrm{MiD} 2 \mathrm{~cm}, \mathrm{MiD} 3 \mathrm{~cm}$, and MiD3l neurons (Fig. $5 D$, 
top left) (O'Malley et al., 1996; Kohashi and Oda, 2008). The activated Mauthner neuron directly activates primary motor neurons ("MN") on the right body side producing an initial contralateral bend ("Bend 1"), whereas the Mauthner plus MiD2cm, $\mathrm{MiD} 3 \mathrm{~cm}$, and $\mathrm{MiD} 3 \mathrm{cl}$ neurons activate a population of yetunidentified contralateral commissural interneurons in the caudal hindbrain and/or spinal cord (Fig. 5D, orange " $\mathrm{X}$ " neurons). With a defined time delay, " $\mathrm{X}$ " neurons directly or indirectly activate motor neurons on the opposite body side producing a counterbend ("Bend 2," Fig. 5D).

In $d c c$ mutants, left-sided head stimuli still activate the contralaterally projecting Mauthner neuron and hence primary motor neurons on the right body side, producing an initial contralateral bend ("Bend 1"). However, the right side " $X$ " neurons are only partially activated because a subset of $\mathrm{MiD} 2 \mathrm{~cm} /$ $\mathrm{MiD} 3 \mathrm{~cm} / \mathrm{MiD} 3 \mathrm{cl}$ neurons now extend ipsilaterally, ectopically synapsing on and activating left side " $\mathrm{X}$ " neurons. This ectopic ipsilateral " $X$ " activation in turn activates right side motor neurons, outcompeting the weakened activation of left side motor neurons, producing a right-sided counterbend ("Bend 2," Fig. $5 D)$. Ablating the inappropriately projecting $\mathrm{MiD} 2 \mathrm{~cm}, \mathrm{MiD} 3 \mathrm{~cm}$, and MiD3l neurons in $\mathrm{dcc}$ mutants removes the conflicting ipsilateral interneuron activation, allowing appropriate counterbend direction (Fig. 5D). Consistent with this model, Mauthner neurons form direct synaptic contacts with trunk motor neurons and spinal interneurons (Myers, 1985; Jontes et al., 2000; Liao and Fetcho, 2008; Satou et al., 2009). Finally, although our data demonstrate that aberrant bilateral connectivity of this small neural array is sufficient to induce inappropriate mirror movement-like behaviors, the direct synaptic targets of $M i D 2 \mathrm{~cm}, \mathrm{MiD} 3 \mathrm{~cm}$, and $\mathrm{MiD} 3 \mathrm{cl}$ have not yet been identified, making their future identification a necessity to further understand how minor changes in circuit connectivity cause dramatic changes in behavior.

\section{Insight from zebrafish on descending control of left/right coordination and MMD}

In human patients, DCC disruption produces mirror movements most noticeably in the hand and fingers, movements thought to be controlled by the "cortico-motoneuronal" subset of corticospinal neurons (Lemon and Griffiths, 2005; Cox et al., 2012; Peng and Charron, 2013). In simpler vertebrates, such as zebrafish, many motor control functions of the human corticospinal tract are instead controlled by reticulospinal tract neurons, representing an analogous yet simplified system in which to study descending motor control (Vulliemoz et al., 2005). Interestingly, corticomotoneuronal neurons form direct synaptic connections with spinal motor neurons (Bortoff and Strick, 1993), akin to the direct motor neuron activation by the Mauthner (Fig. 5D) (Myers, 1985; Jontes et al., 2000; Chong and Drapeau, 2007). The Mauthner and $\mathrm{MiD} 2 \mathrm{~cm} / \mathrm{MiD} 3 \mathrm{~cm} / \mathrm{MiD} 31$ neurons comprise a commissural reticulospinal hindbrain array controlling left/right coordination of body movements (Nissanov et al., 1990; O'Malley et al., 1996; Liu and Fetcho, 1999). Therefore, we focused on the behavioral consequences of ectopic bilateral descending $\mathrm{MiD} 2 \mathrm{~cm} / \mathrm{MiD} 3 \mathrm{~cm} / \mathrm{MiD} 3 \mathrm{cl}$ axonal projections caused by $d c c$ mutation.

Unlike humans, where right and left limbs can move independently or in concert, zebrafish spinal motor circuit organization precludes simultaneous bilateral trunk contraction (Granato et al., 1996; Drapeau et al., 2002; Hirata et al., 2005). Whereas inappropriate bilateral corticospinal input in humans with DCC disruption produces an involuntary mirror movement on the incorrect side simultaneous with the intended movement, de- scending bilateral activation in zebrafish must resolve into unilateral body bends. Therefore, we expect analogous mirror movement-like defects in zebrafish $d c c$ mutants to instead manifest as delays and/or randomization of the left/right direction of lateralized body bends. During touch-evoked startle responses of $d c c$ mutants, we frequently observe counterbends performed in the same direction as the initial bends (Fig. $3 B-D$ ). Like human mirror movements, these mirror movement-like bends inappropriately occur on the opposite side from the "intended" stereotyped counterbend and are largely the result of descending motor control defects (Fig. 5C). Thus, the ability to focus on a simplified hindbrain circuit and stereotyped movement patterns in zebrafish allows us to elucidate a basic function of $d c c$-dependent motor circuits: modeling not the exact mirror movements of limbs, but rather the conserved requirement of $d c c$ in descending left/right motor control.

Finally, some individuals with MMD exhibit both bilateral activity downstream of unilateral corticospinal tract activation and inappropriate bilateral motor cortex activity, suggesting that multiple spatially distinct neuronal defects may together produce the overall array of behavioral movement defects (Papadopoulou et al., 2010). Similarly, ablating the misprojecting bilateral $\mathrm{MiD} 2 \mathrm{~cm} / \mathrm{MiD} 3 \mathrm{~cm} / \mathrm{MiD} 3 \mathrm{cl}$ reticulospinal neurons in zebrafish $d c c$ mutants is not sufficient to completely eliminate the mirror movement-like bend defect (Fig. 5C). Furthermore, although $\mathrm{MiD} 2 \mathrm{~cm} / \mathrm{MiD} 3 \mathrm{~cm}$ activity is likely dispensable for spontaneous, acoustically evoked, and tail touch-evoked startle behavior, $d c c$ mutants display counterbend initiation and/or direction defects in these contexts (Figs. 2E-G and 3B) (Liu and Fetcho, 1999; Burgess and Granato, 2007a). Thus, similar to humans, zebrafish require DCC in both the descending $\mathrm{M}$-cell array and additional neurons to initiate and/or maintain left/right coordinated movements. The spontaneous swim bend alternation defects suggest that commissural spinal CPG interneurons may be disrupted in $d c c$ larvae, analogous to the local DCC-dependent neurons coordinating left/right alternating spinal activity in mice (Rabe et al., 2009; Rabe Bernhardt et al., 2012). Identifying these additional DCC-dependent neural circuits regulating left/right alternation will reveal additional mechanisms for how DCC disruption impacts motor behavior.

\section{References}

Bennett KL, Bradshaw J, Youngman T, Rodgers J, Greenfield B, Aruffo A, Linsley PS (1997) Deleted in colorectal carcinoma (DCC) binds heparin via its fifth fibronectin type III domain. J Biol Chem 272:26940-26946. CrossRef Medline

Bhatt DH, McLean DL, Hale ME, Fetcho JR (2007) Grading movement strength by changes in firing intensity versus recruitment of spinal interneurons. Neuron 53:91-102. CrossRef Medline

Bortoff GA, Strick PL (1993) Corticospinal terminations in two new-world primates: further evidence that corticomotoneuronal connections provide part of the neural substrate for manual dexterity. J Neurosci 13:51055118. Medline

Burgess A, Vigneron S, Brioudes E, Labbé JC, Lorca T, Castro A (2010) Loss of human Greatwall results in G2 arrest and multiple mitotic defects due to deregulation of the cyclin B-Cdc2/PP2A balance. Proc Natl Acad Sci U S A 107:12564-12569. CrossRef Medline

Burgess HA, Granato M (2007a) Sensorimotor gating in larval zebrafish. J Neurosci 27:4984-4994. CrossRef Medline

Burgess HA, Granato M (2007b) Modulation of locomotor activity in larval zebrafish during light adaptation. J Exp Biol 210:2526-2539. CrossRef Medline

Burgess HA, Granato M (2008) The neurogenetic frontier: lessons from misbehaving zebrafish. Brief Funct Genomic Proteomic 7:474-482. CrossRef Medline

Burgess HA, Johnson SL, Granato M (2009) Unidirectional startle re- 
sponses and disrupted left-right co-ordination of motor behaviors in robo3 mutant zebrafish. Genes Brain Behav 8:500-511. CrossRef Medline

Chong M, Drapeau P (2007) Interaction between hindbrain and spinal networks during the development of locomotion in zebrafish. Dev Neurobiol 67:933-947. CrossRef Medline

Cincotta M, Borgheresi A, Balzini L, Vannucchi L, Zeloni G, Ragazzoni A, Benvenuti F, Zaccara G, Arnetoli G, Ziemann U (2003) Separate ipsilateral and contralateral corticospinal projections in congenital mirror movements: neurophysiological evidence and significance for motor rehabilitation. Mov Disord 18:1294-1300. CrossRef Medline

Coonan JR, Greferath U, Messenger J, Hartley L, Murphy M, Boyd AW, Dottori M, Galea MP, Bartlett PF (2001) Development and reorganization of corticospinal projections in EphA4 deficient mice. J Comp Neurol 436:248-262. CrossRef Medline

Cox BC, Cincotta M, Espay AJ (2012) Mirror movements in movement disorders: a review. Tremor Other Hyperkinet Mov (N Y) 2.

Depienne C, Cincotta M, Billot S, Bouteiller D, Groppa S, Brochard V, Flamand C, Hubsch C, Meunier S, Giovannelli F, Klebe S, Corvol JC, Vidailhet M, Brice A, Roze E (2011) A novel DCC mutation and genetic heterogeneity in congenital mirror movements. Neurology 76:260-264. CrossRef Medline

Dottori M, Hartley L, Galea M, Paxinos G, Polizzotto M, Kilpatrick T, Bartlett PF, Murphy M, Köntgen F, Boyd AW (1998) EphA4 (Sek1) receptor tyrosine kinase is required for the development of the corticospinal tract. Proc Natl Acad Sci U S A 95:13248-13253. CrossRef Medline

Drapeau P, Saint-Amant L, Buss RR, Chong M, McDearmid JR, Brustein E (2002) Development of the locomotor network in zebrafish. Prog Neurobiol 68:85-111. CrossRef Medline

Fawcett JP, Georgiou J, Ruston J, Bladt F, Sherman A, Warner N, Saab BJ, Scott R, Roder JC, Pawson T (2007) Nck adaptor proteins control the organization of neuronal circuits important for walking. Proc Natl Acad Sci U S A 104:20973-20978. CrossRef Medline

Fazeli A, Dickinson SL, Hermiston ML, Tighe RV, Steen RG, Small CG, Stoeckli ET, Keino-Masu K, Masu M, Rayburn H, Simons J, Bronson RT, Gordon JI, Tessier-Lavigne M, Weinberg RA (1997) Phenotype of mice lacking functional Deleted in colorectal cancer (Dcc) gene. Nature 386: 796-804. CrossRef Medline

Fero K, Yokogawa T, Burgess HA, Kalueff AV (2011) The behavioral repertoire of larval zebrafish. Neuromethods 52:249-292. CrossRef

Finger JH, Bronson RT, Harris B, Johnson K, Przyborski SA, Ackerman SL (2002) The netrin 1 receptors Unc5h3 and Dcc are necessary at multiple choice points for the guidance of corticospinal tract axons. J Neurosci 22:10346-10356. Medline

Fothergill T, Donahoo ALS, Douglass A, Zalucki O, Yuan J, Shu T, Goodhill GJ, Richards LJ (2013) Netrin-DCC signaling regulates corpus callosum formation through attraction of pioneering axons and by modulating Slit2-mediated repulsion. Cereb Cortex. Advance online publication. Retrieved Jan. 17, 2013. doi: 10.1093/cercor/bhs395. CrossRef Medline

Fricke C, Chien CB (2005) Cloning of full-length zebrafish dcc and expression analysis during embryonic and early larval development. Dev Dyn 234:732-739. CrossRef Medline

Gahtan E, Sankrithi N, Campos JB, O’Malley DM (2002) Evidence for a widespread brain stem escape network in larval zebrafish. J Neurophysiol 87:608-614. Medline

Galléa C, Popa T, Billot S, Méneret A, Depienne C, Roze E (2011) Congenital mirror movements: a clue to understanding bimanual motor control. J Neurol 258:1911-1919. CrossRef Medline

Geisbrecht BV, Dowd KA, Barfield RW, Longo PA, Leahy DJ (2003) Netrin binds discrete subdomains of DCC and UNC5 and mediates interactions between DCC and heparin. J Biol Chem 278:32561-32568. CrossRef Medline

Geisler R, Rauch GJ, Baier H, van Bebber F, Bross L, Dekens MP, Finger K, Fricke C, Gates MA, Geiger H, Geiger-Rudolph S, Gilmour D, Glaser S, Gnügge L, Habeck H, Hingst K, Holley S, Keenan J, Kirn A, Knaut H, et al. (1999) A radiation hybrid map of the zebrafish genome. Nat Genet 23: 86-89. CrossRef Medline

Geisler R, Rauch GJ, Geiger-Rudolph S, Albrecht A, van Bebber F, Berger A, Busch-Nentwich E, Dahm R, Dekens MP, Dooley C, Elli AF, Gehring I, Geiger H, Geisler M, Glaser S, Holley S, Huber M, Kerr A, Kirn A, Knirsch $\mathrm{M}$, et al. (2007) Large-scale mapping of mutations affecting zebrafish development. BMC Genomics 8:11. CrossRef Medline

Gordon LR, Gribble KD, Syrett CM, Granato M (2012) Initiation of synapse formation by Wnt-induced MuSK endocytosis. Development 139:10231033. CrossRef Medline

Granato M, van Eeden FJ, Schach U, Trowe T, Brand M, Furutani-Seiki M, Haffter P, Hammerschmidt M, Heisenberg CP, Jiang YJ, Kane DA, Kelsh RN, Mullins MC, Odenthal J, Nüsslein-Volhard C (1996) Genes controlling and mediating locomotion behavior of the zebrafish embryo and larva. Development 123:399-413. Medline

Gyda M, Wolman M, Lorent K, Granato M (2012) The tumor suppressor gene retinoblastoma-1 is required for retinotectal development and visual function in zebrafish. PLoS Genet 8:e1003106. CrossRef Medline

Hatta K (1992) Role of the floor plate in axonal patterning in the zebrafish CNS. Neuron 9:629-642. CrossRef Medline

Hirata H, Saint-Amant L, Downes GB, Cui WW, Zhou W, Granato M, Kuwada JY (2005) Zebrafish bandoneon mutants display behavioral defects due to a mutation in the glycine receptor beta-subunit. Proc Natl Acad Sci U S A 102:8345-8350. CrossRef Medline

Jao LE, Maddison L, Chen W, Burgess SM (2008) Using retroviruses as a mutagenesis tool to explore the zebrafish genome. Brief Funct Genomic Proteomic 7:427-443. CrossRef Medline

Jen JC, Chan WM, Bosley TM, Wan J, Carr JR, Rüb U, Shattuck D, Salamon G, Kudo LC, Ou J, Lin DD, Salih MA, Kansu T, Al Dhalaan H, Al Zayed Z, MacDonald DB, Stigsby B, Plaitakis A, Dretakis EK, Gottlob I, et al. (2004) Mutations in a human ROBO gene disrupt hindbrain axon pathway crossing and morphogenesis. Science 304:1509-1513. CrossRef Medline

Jontes JD, Buchanan J, Smith SJ (2000) Growth cone and dendrite dynamics in zebrafish embryos: early events in synaptogenesis imaged in vivo. Nat Neurosci 3:231-237. CrossRef Medline

Keino-Masu K, Masu M, Hinck L, Leonardo ED, Chan SS, Culotti JG, TessierLavigne M (1996) Deleted in Colorectal Cancer (DCC) encodes a netrin receptor. Cell 87:175-185. CrossRef Medline

Kent WJ (2002) BLAT: the BLAST-like alignment tool. Genome Res 12: 656-664. CrossRef Medline

Kimmel CB, Patterson J, Kimmel RO (1974) The development and behavioral characteristics of the startle response in the zebra fish. Dev Psychobiol 7:47-60. CrossRef Medline

Kohashi T, Oda Y (2008) Initiation of Mauthner- or non-Mauthnermediated fast escape evoked by different modes of sensory input. J Neurosci 28:10641-10653. CrossRef Medline

Koyama M, Kinkhabwala A, Satou C, Higashijima S, Fetcho J (2011) Mapping a sensory-motor network onto a structural and functional ground plan in the hindbrain. Proc Natl Acad Sci U S A 108:1170-1175. CrossRef Medline

Kruger RP, Lee J, Li W, Guan KL (2004) Mapping netrin receptor binding reveals domains of Unc5 regulating its tyrosine phosphorylation. J Neurosci 24:10826-10834. CrossRef Medline

Kullander K, Butt SJ, Lebret JM, Lundfald L, Restrepo CE, Rydström A, Klein R, Kiehn O (2003) Role of EphA4 and EphrinB3 in local neuronal circuits that control walking. Science 299:1889-1892. CrossRef Medline

Lemon RN, Griffiths J (2005) Comparing the function of the corticospinal system in different species: organizational differences for motor specialization? Muscle Nerve 32:261-279. CrossRef Medline

Lepage JF, Beaulé V, Srour M, Rouleau G, Pascual-Leone A, Lassonde M, Théoret H (2012) Neurophysiological investigation of congenital mirror movements in a patient with agenesis of the corpus callosum. Brain Stimul 5:137-140. CrossRef Medline

Liao JC, Fetcho JR (2008) Shared versus specialized glycinergic spinal interneurons in axial motor circuits of larval zebrafish. J Neurosci 28: 12982-12992. CrossRef Medline

Liu KS, Fetcho JR (1999) Laser ablations reveal functional relationships of segmental hindbrain neurons in zebrafish. Neuron 23:325-335. CrossRef Medline

Lorent K, Liu KS, Fetcho JR, Granato M (2001) The zebrafish space cadet gene controls axonal pathfinding of neurons that modulate fast turning movements. Development 128:2131-2142. Medline

McClenahan P, Troup M, Scott EK (2012) Fin-tail coordination during escape and predatory behavior in larval zebrafish. PLoS One 7:e32295. CrossRef Medline

Metcalfe WK, Mendelson B, Kimmel CB (1986) Segmental homologies among reticulospinal neurons in the hindbrain of the zebrafish larva. J Comp Neurol 251:147-159. CrossRef Medline

Michelmore RW, Paran I, Kesseli RV (1991) Identification of markers 
linked to disease-resistance genes by bulked segregant analysis: a rapid method to detect markers in specific genomic regions by using segregating populations. Proc Natl Acad Sci U S A 88:9828-9832. CrossRef Medline

Myers PZ (1985) Spinal motoneurons of the larval zebrafish. J Comp Neurol 236:555-561. CrossRef Medline

Neff MM, Turk E, Kalishman M (2002) Web-based primer design for single nucleotide polymorphism analysis. Trends Genet 18:613-615. CrossRef Medline

Nissanov J, Eaton RC, DiDomenico R (1990) The motor output of the Mauthner cell, a reticulospinal command neuron. Brain Res 517:88-98. CrossRef Medline

O’Malley DM, Kao YH, Fetcho JR (1996) Imaging the functional organization of zebrafish hindbrain segments during escape behaviors. Neuron 17:1145-1155. CrossRef Medline

Papadopoulou M, Chairopoulos K, Anagnostou E, Kokotis P, Zambelis T, Karandreas N (2010) Concurrent bilateral projection and activation of motor cortices in a patient with congenital mirror movements: a TMS study. Clin Neurol Neurosurg 112:824-828. CrossRef Medline

Peng J, Charron F (2012) Lateralization of motor control in the human nervous system: genetics of mirror movements. Curr Opin Neurobiol 23:109-118. CrossRef Medline

Rabe Bernhardt N, Memic F, Gezelius H, Thiebes AL, Vallstedt A, Kullander K (2012) DCC mediated axon guidance of spinal interneurons is essential for normal locomotor central pattern generator function. Dev Biol 366:279-289. CrossRef Medline

Rabe N, Gezelius H, Vallstedt A, Memic F, Kullander K (2009) Netrin-1dependent spinal interneuron subtypes are required for the formation of left-right alternating locomotor circuitry. J Neurosci 29:15642-15649. CrossRef Medline
Rauch GJ, Granato M, Haffter P (1997) A polymorphic zebrafish line for genetic mapping using SSLPs on high-percentage agarose gels. Technical Tips Online 2:148-150. CrossRef

Satou C, Kimura Y, Kohashi T, Horikawa K, Takeda H, Oda Y, Higashijima S (2009) Functional role of a specialized class of spinal commissural inhibitory neurons during fast escapes in zebrafish. J Neurosci 29:6780-6793. CrossRef Medline

Schindelin J, Arganda-Carreras I, Frise E, Kaynig V, Longair M, Pietzsch T, Preibisch S, Rueden C, Saalfeld S, Schmid B, Tinevez JY, White DJ, Hartenstein V, Eliceiri K, Tomancak P, Cardona A (2012) Fiji: an opensource platform for biological-image analysis. Nat Methods 9:676-682. CrossRef Medline

Serafini T, Colamarino SA, Leonardo ED, Wang H, Beddington R, Skarnes WC, Tessier-Lavigne M (1996) Netrin-1 is required for commissural axon guidance in the developing vertebrate nervous system. Cell 87:10011014. CrossRef Medline

Srour M, Rivière JB, Pham JM, Dubé MP, Girard S, Morin S, Dion PA, Asselin G, Rochefort D, Hince P, Diab S, Sharafaddinzadeh N, Chouinard S, Théoret H, Charron F, Rouleau GA (2010) Mutations in DCC cause congenital mirror movements. Science 328:592. CrossRef Medline

Vulliemoz S, Raineteau O, Jabaudon D (2005) Reaching beyond the midline: why are human brains cross wired? Lancet Neurol 4:87-99. CrossRef Medline

Waskiewicz AJ, Rikhof HA, Hernandez RE, Moens CB (2001) Zebrafish Meis functions to stabilize $\mathrm{Pbx}$ proteins and regulate hindbrain patterning. Development 128:4139-4151. Medline

Wolman MA, Jain RA, Liss L, Granato M (2011) Chemical modulation of memory formation in larval zebrafish. Proc Natl Acad Sci U S A 108: 15468-15473. CrossRef Medline 